\title{
Evaluation of Treatment for Volumetric Muscle Loss in a Porcine Tibia Segmental Bone Defect Model
}

Nicklaus Diggins ${ }^{1}$, Seungyup Sun ${ }^{1}$, Benjamin T. Corona ${ }^{2}$, Aamir Tucker ${ }^{1}$, Alex Brinker ${ }^{1}$, Michael Savaglio ${ }^{1}$, Gremah Adam ${ }^{1}$, Zachary Gunderson ${ }^{1}$, Roman M. Natoli ${ }^{1}$, Todd O. McKinley ${ }^{1}$, Melissa A. Kacena ${ }^{1}$

${ }^{1}$ Department of Orthopaedic Surgery, Indiana University School of Medicine, Indiana, USA, 'Wake Forest School of Medicine, North Carolina, USA

Background and Hypothesis: Injury to soft tissue with volumetric muscle loss (VML) concomitant to segmental bone defects (SBDs) can be difficult to treat. One potential option is skeletal muscle autograft (SMA). We hypothesize the maximal torque measured in vivo and Radiographic Union Scale for Tibial Fractures (RUST) scores will be increased in the VML+SBD+SMA group compared to the VML+SBD group, but will be decreased compared to SBD alone.

Experimental Design or Project Methods: 18 male, Yucatan minipigs aged 1821 months were randomized into 3 groups: $V M L+S B D+S M A, V M L+S B D$, and SBD. This study is still ongoing with 12 pigs having had the surgery performed to date. In vivo muscle testing was performed prior to surgery in both hindlimbs to assess baseline strength. RUST scores and in vivo muscle testing was/will be performed at 1, 2, and 3 months post-injury. All procedures were conducted following approved IACUC protocol.

Results: Muscle testing results demonstrate no significant difference between control and operative limbs pre-surgery (T-tests, $p=0.673,0.824$, and 0.739 for $\mathrm{VML}+\mathrm{SBD}+\mathrm{SMA}, \mathrm{VML}+\mathrm{SBD}$ and SBD groups, respectively), or between the 3 different groups pre- or post-surgery (2-way ANOVA, $p=0.788$ ). The average RUST score for each treatment group at 2 months was $6.8 \pm 2.2,7.77 .7 \pm 1.1$, and 8.6 8.6 \pm 0.4 for $V M L+S B D+S M A(n=2), V M L+S B D(n=5)$, and SBD $(n=3)$ groups, respectively.

Conclusion and Potential Impact: The lack of difference amongst groups on pre-operative muscle testing and a difference post-operatively between control and operative legs post-operatively help to validate this study. Preliminary results demonstrate a reduced RUST score for the VML+SBD+SMA group; however, this along with the lack of difference found in muscle testing results postoperatively may be an artifact due to small sample size and/or timing. Development of a model for VML and SBD will allow for testing of therapies for treat clinical problems. 